\title{
Nonhormonal Hot Flash Management for Breast Cancer Survivors: A Systematic Review and Network Meta-Analysis
}

\author{
Jian Liu, ${ }^{1}$ Guangning Nie, ${ }^{1}$ Yang Li, ${ }^{1}$ Zehuai Wen $\left(\mathbb{D},{ }^{2}\right.$ Liming Lu $\mathbb{D},{ }^{3}$ Li Xie ${ }^{\mathbb{D}},{ }^{4}$ \\ Dongdong Cao, ${ }^{5}$ Yafang Lai, ${ }^{6}$ and Hongyan Yang $\mathbb{D}^{1,7}$ \\ ${ }^{1}$ Department of Gynecology, The Second Affiliated Hospital of Guangzhou University of Chinese Medicine, \\ Dade Road, Yuexiu District, Guangzhou 510120, Guangdong, China \\ ${ }^{2}$ Key Unit of Methodology in Clinical Research, The Second Affiliated Hospital of Guangzhou University of Chinese Medicine, \\ Guangzhou, Guangdong, China \\ ${ }^{3}$ Clinical Research Center, South China Research Center for Acupuncture and Moxibustion, \\ Medical College of Acu-Moxi and Rehabilitation, Guangzhou University of Chinese Medicine, Guangzhou, Guangdong, China \\ ${ }^{4}$ The Second Clinical College of Guangzhou University of Chinese Medicine, Guangzhou, Guangdong, China \\ ${ }^{5}$ Department of Chinese Medicine, Affiliated Hospital of Guilin Medical University, Guilin, Guangxi, China \\ ${ }^{6}$ Department of Gynecology, Foshan Hospital of Traditional Chinese Medicine, Guangzhou, Guangdong, China \\ ${ }^{7}$ Guangdong Provincial Key Laboratory of Clinical Research on Traditional Chinese Medicine Syndrome, \\ The Second Affiliated Hospital of Guangzhou University of Chinese Medicine, Guangzhou, Guangdong, China
}

Correspondence should be addressed to Hongyan Yang; hongyanyang@gzucm.edu.cn

Received 26 November 2019; Revised 26 February 2020; Accepted 28 February 2020; Published 28 April 2020

Academic Editor: Oliver Micke

Copyright (C) 2020 Jian Liu et al. This is an open access article distributed under the Creative Commons Attribution License, which permits unrestricted use, distribution, and reproduction in any medium, provided the original work is properly cited.

\begin{abstract}
Aim of the Study. Hot flashes impair the quality of life of breast cancer survivors. Nonhormonal management is an important consideration. The objective of this network meta-analysis (NMA) is to compare the therapeutic efficacy and safety of nonhormonal hot flash treatments for breast cancer survivors. Materials and Methods. We conducted a systematic literature search in PubMed, Cochrane Central Register of Controlled Trials, Embase, Chinese Biomedicine Database (CBM), China National Knowledge Infrastructure (CNKI), Wan Fang, and VIP up to May 2018. Randomized controlled trials (RCTs) reporting nonhormonal hot flash treatments for breast cancer survivors were included. Primary outcome measurements were hot flash frequency and hot flash score of posttreatment. The methodological quality of each study was assessed with Cochrane's risk of bias tool. Results. 16 RCTs involving 2,349 participants were included. The nonhormonal therapies used in the included studies were classified as follows: lifestyle changes, mind-body techniques, dietary/supplements, SSRIs/SNRIs, other medications, and other therapies. Pairwise meta-analysis showed that the general effect of nonhormonal management was statistically more effective than no treatment/placebo/sham in reducing hot flash frequency (SMD $=-0.60,95 \% \mathrm{CI}[-1.13$, $-0.06] ; P=0.03)$ ) and hot flash score (SMD $=-0.38,95 \%$ CI $[-0.68,-0.08])$. For hot flash frequency, results from the NMA showed that there was no statistically significant difference between any two of the nonhormonal treatments. Another NMA result indicated that acupuncture (other therapies) was 16.05 points more effective in reducing hot flash scores than no treatment/waitlist $(\mathrm{SMD}=-16.05,95 \% \mathrm{CI}[-30.2,-1.99])$. These results were statistically significant. Acupuncture was also ranked the optimal nonhormonal therapy for both hot flash frequency and hot flash score. The safety analysis showed that there were few related adverse events during acupuncture and that drug related adverse reactions could have also occurred in studies using drug interventions Conclusions. This network meta-analysis comparing nonhormonal treatments suggested that acupuncture might be more effective in improving hot flashes for breast cancer survivors. A pronounced placebo response was found during hot flash treatments. The evidence of safety for nonhormonal therapies was also insufficient. Therefore, at present, we cannot make confirmative recommendations of nonhormonal hot flash management for breast cancer survivors. This study is registered with PROSPERO (CRD42018082008).
\end{abstract}




\section{Introduction}

Hot flashes are common among breast cancer survivors [1]. This population has more severe and persistent hot flashes due to premature menopause resulting from chemotherapy and the use of endocrine therapies such as tamoxifen and aromatase inhibitors $[2,3]$. It has been demonstrated that $72 \%$ of tamoxifen recipients and $78 \%$ of chemotherapy recipients experience hot flashes [4]. Breast cancer survivors who experience hot flashes also tend to experience more fatigue, worse sleep quality, and lower quality of life [5-7].

Because of concerns that estrogen exposure increases cancer recurrence rates, menopausal hormonal therapy (HT) is not the recommended first-line hot flash treatment for breast cancer survivors [8]. This leaves fewer hot flash treatment options than women without a history of breast cancer. Hence, nonhormonal medicinal treatments (e.g., antidepressants) [9, 10], physical/behavioral treatments (e.g., yoga/exercise, cognitive behavioral therapy) [11, 12], acupuncture $[13,14]$, and natural health products (e.g., black cohosh, soy) $[15,16]$ have been used for hot flashes. Several systematic reviews have summarized studies and data comparing these interventions, both for placebos and for controls [17-20], and have provided favorable recommendations of particular interventions.

The 2015 position statement of the North American Menopause Society [21] indicated that nonhormonal management is now a realistic option for women who cannot take estrogen. In this statement, nonhormonal management of menopause-associated hot flashes is classified into the following categories: lifestyle changes, mindbody techniques, dietary management and supplements, SSRIs/SNRIs, other medications, and other therapies. For clinical decision making, all potential interactions with management treatments need to be weighed against potential effectiveness. While past reviews have considered some nonhormonal treatments in isolation [22], we performed a network meta-analysis $[23,24]$ to compare many alternatives in a unified analysis. This study may provide data to guide physician and patient choice of intervention by efficacy or by side-effect profile.

\section{Methods}

2.1. Protocol and Registration. This research followed the Preferred Reporting Items for Systematic Reviews and MetaAnalyses for Network Meta-Analysis (PRISMA-NMA) checklist [25]. The protocol was registered on the International Prospective Register of Systematic Reviews (PROSPERO; registration number: CRD42018082008).

2.2. Inclusion and Exclusion Criteria. We looked for the following inclusion criteria: (1) participants: women who had been diagnosed with breast cancer and who were experiencing hot flashes, without restrictions on age or cancer stage; (2) intervention: nonhormonal therapies (classified by the 2015 position statement of the North American
Menopause Society [21] including lifestyle changes, mindbody techniques, dietary/supplements, SSRIs/SNRIs, other medications, and other therapies; (3) comparison: hormonal therapies, no treatment/waitlist, placebo/sham treatment; (4) outcomes: hot flash frequency or hot flash scores (frequency $\times$ mean severity or measured using scales such as the hot flash diary) of posttreatment, adverse events; (5) study design: randomized clinical trial (RCT); (6) language: English or Chinese studies.

We excluded studies based on the following criteria: (1) participants with multiple forms of cancer; (2) participants who had not received nonhormonal therapy; (3) self-control and non-RCTs; (4) preclinical studies, systematic reviews, case reports, and meta-analyses; (5) protocols, unpublished or duplicate studies.

2.3. Information Sources and Search Strategy. We systematically searched the following databases: PubMed, Cochrane Central Register of Controlled Trials, Embase, Chinese Biomedicine Database (CBM), China National Knowledge Infrastructure (CNKI), Wan Fang, and VIP. We identified articles published from journal inception to May 2018, with a limit to studies of RCTs and without limitations on language or publishing format. A more detailed description of the search strategies is presented in Supplementary Material 1.

2.4. Study Selection. Two authors of the review (YL and DDC) independently identified relevant research based on titles and abstracts. Full-text articles were then scanned by four researchers to identify eligible studies. All disagreements were resolved by consensus and adjudicated by a third reviewer (GNN), if necessary. In cases of duplicate citations, the most updated studies were selected for data extraction.

2.5. Data Extraction. According to a standard data collection sheet, two reviewers (LX and YFL) independently extracted data on each included article. Extracted data included year of publication, author information, patient characteristics, sample size, arms of interest, interventions, outcomes, and adverse effect measures and results. In some trials, the data of posttreatment were not given. We used the methods recommended in the Cochrane Handbook for Systematic Reviews of Interventions (version 5.1) [26] to resolve it. Information from studies identified in other reviews was subsequently cross-checked. Inconsistencies and uncertainties were resolved by consultation with a third reviewer (GNN).

2.6. Quality Assessment. Two of the authors (JL, GNN) independently used the Cochrane risk of bias tool, as described in the Cochrane Handbook for Systematic Reviews of Interventions (available at: http://handbook.cochrane. org/) to assess RCT quality. Bias risks for each study were assessed based on 6 factors: random sequence generation, 
allocation concealment, blinding (performance bias, detection bias), incomplete outcome data (attrition bias), selective reporting (reporting bias), and other bias. They were also ranked as either high risk, low risk, or unclear risk.

\subsection{Data Synthesis and Analysis}

2.7.1. Direct Pairwise Meta-Analysis. We first used Review Manager version 5.3 (Cochrane Collaboration, Oxford, United Kingdom) to pool the data. Mean difference (MD) with 95\% confidence intervals (CI) was used to analyze continuous data. The standardized mean difference (SMD) was used when the measurement scales between the different studies could not be corrected by conversion into a single unit of measurement, suggesting an error in the unit reported or a difference in the methods used for measurements [27]. We used the data of posttreatment for analysis. For studies that collected data on a series of time points, only the data from the first time point (immediately after intervention) was included in meta-analysis. Review Manager was also used to perform standard pairwise meta-analysis. We calculated an $I$-square $\left(I^{2}\right)$ test to assess heterogeneity among RCTs [28]. When $I^{2}>50 \%$, they were analyzed using a random-effects model; otherwise, a fixed-effects model was used. Subgroup analyses were conducted according to the type of nonhormonal therapies and the treatment given to the control group. Predefined subgroup analysis and sensitivity analysis were performed regardless of the value of $I^{2}$. We generated forest plots to illustrate the relative strength of curative effects. $P$ value less than 0.05 was deemed statistically significant.

2.7.2. Network Meta-Analysis. We conducted a network meta-analysis (NMA) to estimate the effect for each class and for each individual intervention using Markov chain Monte Carlo methods implemented in WinBUGS (version 1.4.3, MRC Biostatistics Unit, Cambridge, UK) [29]. Two chains with different initial values were run simultaneously to assess convergence using Brooks-Gelman-Rubin diagnostic plots. We utilized the Markov chains for 50,000 simultaneous iterations after the first 5,000 iterations were discarded because they may have had an influence on the arbitrary values. We decided whether to use a fixed-effects or a random-effects approach based on model fit statistics and deviance information criteria (DIC) [30], as well as the amount of heterogeneity present in the pairwise meta-analyses. A model with lower DIC values was preferred, with differences of 3 or more units considered meaningful. If 2 models had similar DIC values, the simplest model (i.e., the fixed-effects model) was preferred. For the network metaanalysis, we assessed the extent to which direct and indirect evidence were consistent, both qualitatively and statistically [30].

\section{Results}

3.1. Study Selection. As shown in Figure 1, a total of 1,563 records were initially identified from all the databases. After removing duplicate publications, 952 studies were left. 879 records remained after scanning the titles and abstracts. 73 of the full-text articles were reviewed for eligibility. 16 trials $[10-16,31-39]$ were included in our final NMA.

3.2. Study Characteristics. The 16 RCTs involving 2,349 participants included in the analyses were published between 2002 and 2016. Participants' ages ranged from 27 to 80 years, while the study sample size ranged from 37 [11] to 422 [33]. Among the included RCTs, there was 1 four-arm trial [14], 3 three-arm trials [33-35], and 12 two-arm trials. Of these studies, $14[10-13,15,16,32-39]$ reported hot flash frequency, while $9[11,12,14,16,31,32,34,35,37]$ reported hot flash scores. The nonhormonal therapies (numbers) of the included studies were classified as follows: lifestyle changes: yoga (1), physical exercise (1); mind-body techniques: hypnosis (1), cognitive behavioral therapy (CBT) (2); dietary/supplements: soy beverages (1), black cohosh (1), melatonin (1), magnesium oxide (1); SSRIs/SNRIs: sertraline (1); other medications: gabapentin (1); other therapies: acupuncture (6). Nonhormonal therapies details, retention time, frequency, and duration of therapy are shown in Table 1.

3.3. Risk of Bias in Included Studies. The methodological quality of the included studies was generally poor. Most studies $(12,75 \%)$ used either a random number table or computer software for sequence generation and had been assessed as low risk of bias. Eight studies were assessed as unclear risk of bias for sequence generation and allocation concealment because there had been no clear description. Participants in seven studies were unlikely to have been blinded, due to the nature of some of the interventions and comparators, and personnel had not been blinded to group allocation. Therefore, these studies were assessed as high risk for blinding of participants and personnel. Nine studies reported that outcome assessments had been evaluated by a third party who had been unaware of the interventions; these were judged as of low risk. All studies were assessed as low risk for incomplete outcome data, as reasons for drop-out were reported, and drop-out numbers were consistent across groups. All but 1 of the studies were assessed as low risk for selective outcome reporting. The 1 exception was assessed as unclear risk because reported trial registration and published protocol cannot be identified. Uncertainty regarding the other bias was due mostly to insufficient reporting. Risk of bias assessments for all the included studies are summarized in Supplementary Material 2.

3.4. Pairwise Meta-Analyses. We performed meta-analyses on the different outcomes for hot flash frequency and hot flash score.

3.5. Hot Flash Frequency. Overall, the 6 nonhormonal therapies mentioned above were more efficacious in reducing hot flash frequency than no treatment/placebo/sham $(\mathrm{SMD}=-0.60, \quad 95 \% \quad \mathrm{CI} \quad[-1.13, \quad-0.06] ; \quad P=0.03)$. 


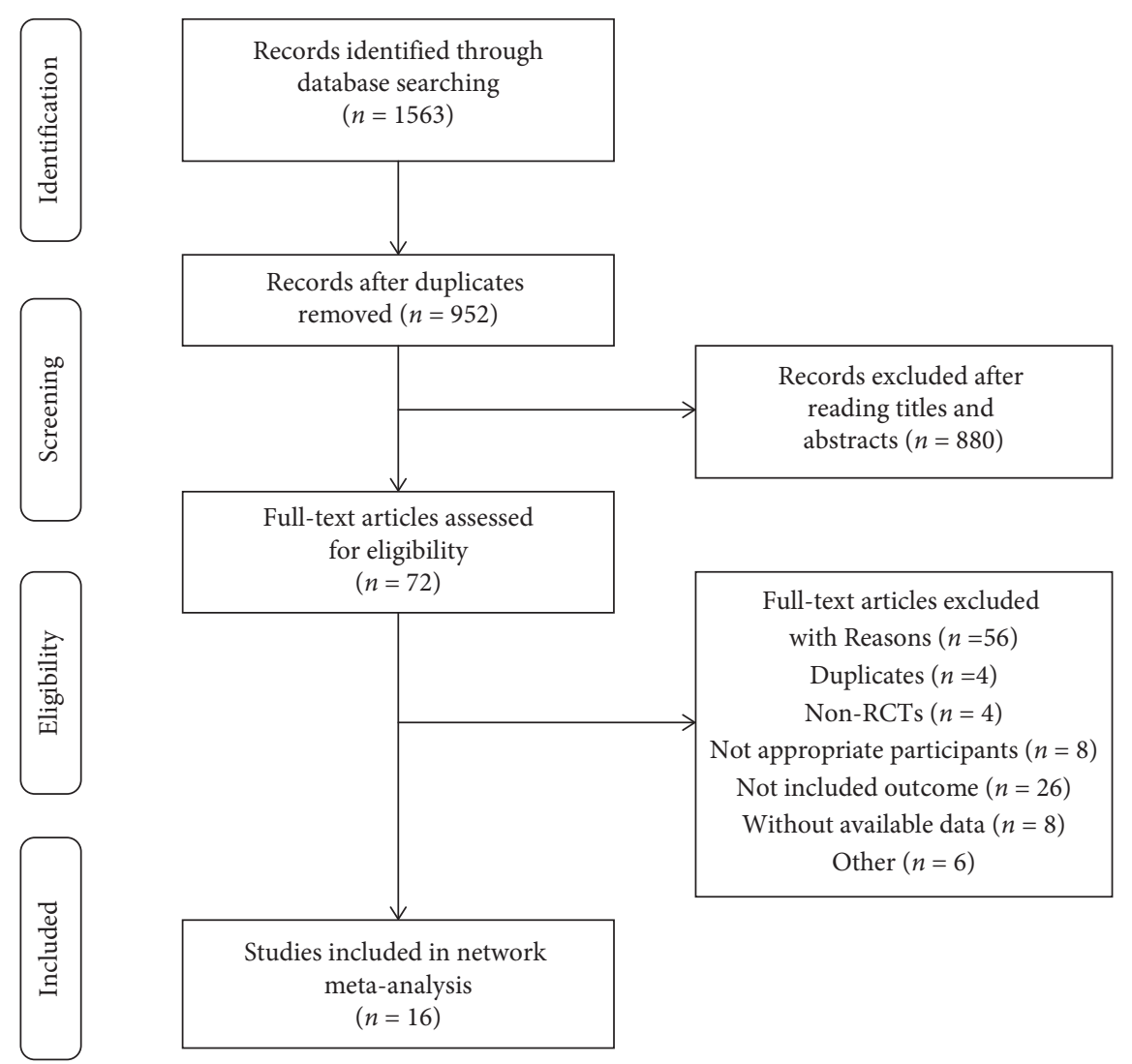

FIgURE 1: PRISMA flowchart.

Acupuncture, classified into other therapies, was 3.92 points less effective in reducing hot flash frequency than HT $(\mathrm{MD}=3.92,95 \% \mathrm{CI}[2.36,5.48], P<0.00001)$. There was no statistically significant difference between mind-body techniques and lifestyle changes $(\mathrm{MD}=-0.32,95 \% \mathrm{CI}$ $[-0.82,0.18])$. The mean, standard deviation (SD), and sample size of the groups are shown in Figure 2.

3.6. Hot Flash Score. Nonhormonal management was significantly more effective than no treatment/placebo/sham $(\mathrm{SMD}=-0.38,95 \% \mathrm{CI}[-0.68,-0.08] ; P=0.01)$. This effect was particularly noticeable for lifestyle changes $(\mathrm{SMD}=-1.49,95 \% \mathrm{CI}[-2.26,-0.72] ; P=0.00002)$, mindbody techniques $(\mathrm{SMD}=-0.94,95 \%$ CI $[-1.55,-0.33]$; $P=0.003)$, and acupuncture $(\mathrm{SMD}=-0.65,95 \%$ CI $[-0.94$, $-0.36] ; P<0.0001)$. There was no statistically significant difference between placebo and the following nonhormonal management treatments: dietary/supplements $(\mathrm{SMD}=-0.01,95 \% \mathrm{CI}[-0.21,0.19])$, other medications $(\mathrm{SMD}=-0.49,95 \% \mathrm{CI}[-1.02,0.03])$. Mean, standard deviation (SD) and sample size of the groups are shown in Figure 3.

\subsection{Network Meta-Analysis}

3.7.1. Hot Flash Frequency. The NMA showed that nonhormonal therapies were more efficacious than no treatment/waitlist, but less effective than HT. Meanwhile, there was no statistically significant difference in hot flash frequency between any two of the treatments.

3.7.2. Hot Flash Score. The results suggest that nonhormonal therapies were more effective than no treatment/waitlist, with statistically significant difference for two of the therapies: other therapies (acupuncture) (SMD $=-16.05,95 \% \mathrm{CI}$ $[-30.2,-1.99])$ and lifestyle changes $(\mathrm{SMD}=-11.28,95 \% \mathrm{CI}$ $[-20.09,-2.52])$. Placebo/sham could decrease hot flash score by 12.27 points more than no treatment/waitlist $(\mathrm{SMD}=12.27,95 \% \mathrm{CI}[-11.99,4.43])$.

The results of the network meta-analysis are shown in Table 2 and the network diagram of the interventions is shown in Figure 4.

\subsection{Ranking}

3.8.1. Hot Flash Frequency. The rankings of the various nonhormonal management interventions are displayed in Table 3. For reducing hot flash frequency of breast cancer survivors, other therapies (acupuncture) were ranked the optimal method, followed by other medications (gabapentin). The remainders were ranked as follows: dietary/supplements, mind-body techniques, lifestyle changes, and SSRIs/SNRIs, which were ranked after placebo/sham.

The ranking of the interventions is shown in Table 3. 
TABLE 1: Characteristics of the 16 included studies.

\begin{tabular}{|c|c|c|c|}
\hline Reference & Participants & Interventions and comparisons & $\begin{array}{l}\text { Outcomes } \\
\text { measures }\end{array}$ \\
\hline $\begin{array}{l}\text { Van Patten } \\
\text { et al. [16] }\end{array}$ & $\begin{array}{l}123 \text { participants ( } 38 \text { on tamoxifen) } \\
\text { (i) Breast cancer stages: I-III } \\
\text { (ii) Menopausal }(\geq 12 \text { months of amenorrhea) } \\
\text { (iii) Not using HRT for } \geq 4 \text { months } \\
\text { (iv) Hot flashes with a score (frequency } \times \text { intensity) } \\
\text { of } \geq 10 / \text { wk }\end{array}$ & $\begin{array}{l}\text { 12-week study } \\
2 \text { groups: } \\
\text { (i) Soy beverage (containing } 90 \mathrm{mg} \text { of } \\
\text { isoflavones), } 500 \mathrm{ml} \text { daily } \\
\quad \text { (ii) Placebo (a rice beverage) }\end{array}$ & (1) + (2) \\
\hline $\begin{array}{l}\text { Jacobson et al. } \\
{[15]}\end{array}$ & $\begin{array}{l}89 \text { participants (59 on tamoxifen) } \\
\text { (i) Age } \geq 18 \\
\text { (ii) Completing primary therapy-before entering } \\
\text { the trial } \geq 2 \text { months }\end{array}$ & $\begin{array}{l}60 \text {-day study } \\
2 \text { groups: } \\
\text { (i) Black cohosh, one tablet twice daily } \\
\text { (ii) Placebo }\end{array}$ & (1) \\
\hline Lesi et al. [31] & $\begin{array}{l}190 \text { participants }(170 \text { on } \mathrm{HT}) \\
\text { (i) Age } 18 \text { to } 65 \text { years } \\
\text { (ii) Spontaneous or induced } \\
\text { (iii) Amenorrhea } \geq 6 \text { months } \\
\text { (iv) Hot flashes mean number } \geq 6\end{array}$ & $\begin{array}{l}\text { 12-week study } \\
2 \text { groups: } \\
\text { (i) Acupuncture once per week } \\
\text { (ii) Waitlist }\end{array}$ & (2) \\
\hline $\begin{array}{l}\text { Hervik et al. } \\
{[13]}\end{array}$ & $\begin{array}{l}95 \text { participants } \\
\text { (i) Tamoxifen used } \geq 3 \text { months } \\
\text { (ii) No menstruation } \geq 3 \text { months }\end{array}$ & $\begin{array}{l}\text { 10-week study } \\
2 \text { groups: } \\
\text { (i) Acupuncture } \\
\text { (ii) Sham acupuncture } \\
\text { Both twice weekly for the first } 5 \text { weeks, once a week } \\
\text { for the following } 5 \text { weeks }\end{array}$ & (1) \\
\hline $\begin{array}{l}\text { Frisk et al. } \\
\text { [36] }\end{array}$ & $\begin{array}{l}45 \text { participants } \\
\text { (i) Breast cancer stages: in situ, T1 and T2 tumors } \\
\text { with four or fewer lymph nodes positive for } \\
\text { metastasis, T3 tumors without metastasis }\end{array}$ & $\begin{array}{l}\text { 12-week study } \\
2 \text { groups: } \\
\text { (i) Electroacupuncture (EA) twice a week for the } \\
\text { first } 2 \text { weeks, and once a week for } 10 \text { weeks } \\
\text { (ii) Hormone therapy (HT) estrogen/ } \\
\text { progestagen combination or continuous combined } \\
\text { estrogen/progestagen }\end{array}$ & (1) \\
\hline Mao et al. [14] & $\begin{array}{l}120 \text { participants } \\
\text { (i) Breast cancer stages: I-III } \\
\text { (ii) Hot flashes each day } \geq 2 \\
\text { (iii) Hot flashes presented } \geq 1 \text { month } \\
\text { (iv) Nonhormonal contraceptives }\end{array}$ & $\begin{array}{l}\text { 8-week study } \\
4 \text { groups: } \\
\text { (i) Electroacupuncture (EA) } \\
\text { (ii) Sham acupuncture } \\
\text { Both twice per week for } 2 \text { weeks, then once per } \\
\text { week for } 6 \text { more weeks } \\
\text { (iii) Gabapentin (GP) } \\
\text { (iv) Placebo } \\
\text { Both one pill ( } 300 \mathrm{mg} \text { ) at bedtime for } 3 \text { days, then } \\
\text { twice per day for } 3 \text { days, and then three times per } \\
\text { day for the remaining } 50 \text { days }\end{array}$ & (2) \\
\hline $\begin{array}{l}\text { Elkins et al. } \\
\text { [37] }\end{array}$ & $\begin{array}{l}51 \text { participants } \\
\text { (i) Age } \geq 18 \\
\text { (ii) Hot flashes per week } \geq 14 \\
\text { (iii) Hot flashes presented } \geq 1 \text { month }\end{array}$ & $\begin{array}{l}\text { 5-week study } \\
2 \text { groups: } \\
\text { (i) Hypnosis intervention five weekly sessions, } \\
\text { each to last approximately } 50 \text { minutes } \\
\text { (ii) No treatment }\end{array}$ & (1) + (2) \\
\hline $\begin{array}{l}\text { Deng et al. } \\
\text { [38] }\end{array}$ & $\begin{array}{l}72 \text { participants } \\
\text { (i) Karnofsky performance score } \geq 60 \\
\text { (ii) Hot flashes each day } \geq 3\end{array}$ & $\begin{array}{l}\text { 4-week study } \\
2 \text { groups: } \\
\quad \text { (i) Acupuncture } \\
\text { (ii) Sham acupuncture } \\
\text { Both twice per week }\end{array}$ & (1) \\
\hline $\begin{array}{l}\text { Liljegren et al. } \\
\text { [39] }\end{array}$ & $\begin{array}{l}74 \text { participants } \\
\text { (i) Vasomotor symptoms questionnaire score } \geq 5 \\
\text { (ii) Tamoxifen used } \geq 2 \text { months }\end{array}$ & $\begin{array}{l}\text { 4-week study } \\
2 \text { groups: } \\
\quad \text { (i) Acupuncture } \\
\text { (ii) Control acupuncture } \\
\text { Both twice per week }\end{array}$ & (1) \\
\hline $\begin{array}{l}\text { Carson et al. } \\
{[11]}\end{array}$ & $\begin{array}{l}37 \text { participants } \\
\text { (i) Breast cancer stages: IA-IIB } \\
\text { (ii) Hot flashes each day, } \geq 2 \\
\text { (ii) No hormone replacement therapy and no } \\
\text { menopausal symptom medications, currently or } \\
\text { within prior } 3 \text { months }\end{array}$ & $\begin{array}{l}\text { 8-week study } \\
2 \text { groups: } \\
\quad \text { (i) Yoga, eight weekly 120-min group classes } \\
\text { (5-10 patients per group) } \\
\text { (ii) Waitlist }\end{array}$ & (1) + (2) \\
\hline
\end{tabular}


TABLE 1: Continued.

\begin{tabular}{|c|c|c|c|}
\hline Reference & Participants & Interventions and comparisons & $\begin{array}{l}\text { Outcomes } \\
\text { measures }\end{array}$ \\
\hline $\begin{array}{l}\text { Duijts et al. } \\
\text { [33] }\end{array}$ & $\begin{array}{l}422 \text { participants } \\
\text { (i) Breast cancer stages: T1-4, N0-1, and M0 } \\
\text { (ii) Age }<50 \text { y } \\
\text { (ii) Premenopausal women } \\
\text { (iii) Receiving adjuvant chemotherapy and/or } \\
\text { hormonal therapy } \\
\text { (iv) Chemotherapy completed during } 4 \text { months to } 5 \\
\text { years } \\
\text { (iv) Hormonal therapy ongoing }\end{array}$ & $\begin{array}{l}\text { 12-week study } \\
3 \text { groups: } \\
\text { (i) Cognitive behavioral therapy (CBT), six } \\
\text { weekly group sessions of } 90 \text { minutes each } \\
\quad \text { (ii) Physical exercise (PE) } \\
\text { 12-week, individually tailored, home-based, self- } \\
\text { directed exercise program of } 2.5 \text { to } 3 \text { hours per } \\
\text { week } \\
\quad \text { (iii) Waiting list }\end{array}$ & (1) \\
\hline $\begin{array}{l}\text { Eleanor } \\
\text { Mann et al. } \\
{[12]}\end{array}$ & $\begin{array}{l}96 \text { participants } \\
\text { (i) Age } \geq 18 \\
\text { (ii) Completed medical treatment for breast cancer } \\
\text { adjuvant endocrine treatment }\end{array}$ & $\begin{array}{l}\text { 9-week study } \\
2 \text { groups: } \\
\text { (i) Cognitive behavioral therapy (CBT) } \\
\text { (ii) Waiting list }\end{array}$ & (1) + (2) \\
\hline $\begin{array}{l}\text { Chen et al. } \\
\text { [32] }\end{array}$ & $\begin{array}{l}95 \text { participants } \\
\text { (i) Breast cancer stages: I-III } \\
\text { (ii) Hormonal therapy at least } 60 \text { days prior to } \\
\text { enrollment }\end{array}$ & $\begin{array}{l}\text { 4-months study } \\
2 \text { groups: } \\
\text { (i) Melatonin } 3 \mathrm{mg} \text { daily } \\
\text { (ii) Placebo }\end{array}$ & (1) + (2) \\
\hline $\begin{array}{l}\text { Park et al. } \\
\text { [34] }\end{array}$ & $\begin{array}{l}358 \text { participants } \\
\text { (i) Postmenopausal women } \\
\text { (ii) Hot flashes each week } \geq 14 \\
\text { (iii) Hot flashes presented for } \geq \text { days }\end{array}$ & $\begin{array}{l}\text { 8-week study } \\
3 \text { groups: } \\
\text { (i) Magnesium oxide } 800 \mathrm{mg} \text { daily } \\
\text { (ii) Magnesium oxide } 1200 \mathrm{mg} \text { daily } \\
\text { (iii) Placebo }\end{array}$ & (1) + (2) \\
\hline $\begin{array}{l}\text { Kimmick } \\
\text { et al. [10] }\end{array}$ & $\begin{array}{l}62 \text { participants } \\
\text { (i) Age } \geq 18 \\
\text { (ii) Breast cancer stages: } 0 \text {-IIIB } \\
\text { (iii) Adjuvant tamoxifen therapy } \\
\text { (iv) Hot flashes per day } \geq 1 \\
\end{array}$ & $\begin{array}{l}\text { 6-week study } \\
2 \text { groups: } \\
\text { (i) Sertraline, } 50 \mathrm{mg} \text { each morning } \\
\text { (ii) Placebo }\end{array}$ & (1) \\
\hline $\begin{array}{l}\text { Pandya et al. } \\
{[35]}\end{array}$ & $\begin{array}{l}420 \text { participants } \\
\text { (i) Age } \geq 18 \\
\text { (ii) Hot flashes each day } \geq 2 \\
\text { (iii) Nonsteroidal contraceptive measures } \\
\text { (iv) Patients currently receiving chemotherapy } \\
\text { were not eligible, although endocrine therapies were } \\
\text { allowed }\end{array}$ & $\begin{array}{l}\text { 8-week study } \\
3 \text { groups: } \\
\text { (i) Gabapentin } 900 \text { daily } \\
\text { (ii) Gabapentin } 300 \text { daily } \\
\text { (iii) Placebo }\end{array}$ & (1) + (2) \\
\hline
\end{tabular}

(1) indicates hot flash frequency. (2) indicates hot flash score.

3.8.2. Hot Flash Score. The rankings of the various nonhormonal treatment methods are displayed in Table 3. In terms of hot flash score, acupuncture (other therapies) was also ranked the optimal therapy, followed by lifestyle changes, other medications, dietary/supplements, and mindbody techniques. The last four methods were also ranked after placebo/sham.

\subsection{Inconsistency Assessment}

3.9.1. Hot Flash Frequency. For the inconsistency test outcome of hot flash frequency, 95\% CI of all loops included 0 , which showed that no significant inconsistency was found. However, other loops (lifestyle changes, mindbody techniques, no treatment/waitlist) were found to have statistical inconsistency between direct and indirect comparisons. The results are shown in Supplementary Material 3.
3.9.2. Hot Flash Score. A Z-test illustrates the inconsistency of the NMA. 95\% CI of all loops included 0 for their hot flash scores, which indicated that no significant inconsistency had been found. Also, there was no loop statistical inconsistency between direct and indirect comparisons.

3.10. Safety. Nine included studies $[11,13,33-37,39]$ reported that no adverse events had been found in either the intervention group or the control group. 8 RCTs $[10,12,14-16,31,32,38]$ reported adverse events. 1 study [15] reported 3 serious adverse events: hysterectomy, breast recurrence, and appendectomy, but these were not related to the intervention medicine black cohosh. 1 study [12] indicated that none of the adverse events were related to CBT. The 2 RCTs $[16,32]$ using dairy interventions (soy beverages, melatonin) showed more digestive system effects than the placebo. 1 study [10] using sertraline (SSRIs) showed more side effects such as nausea and diarrhea than the placebo did. 
1.1. Non-hormonal therapies vs no treatment/placebo/sham

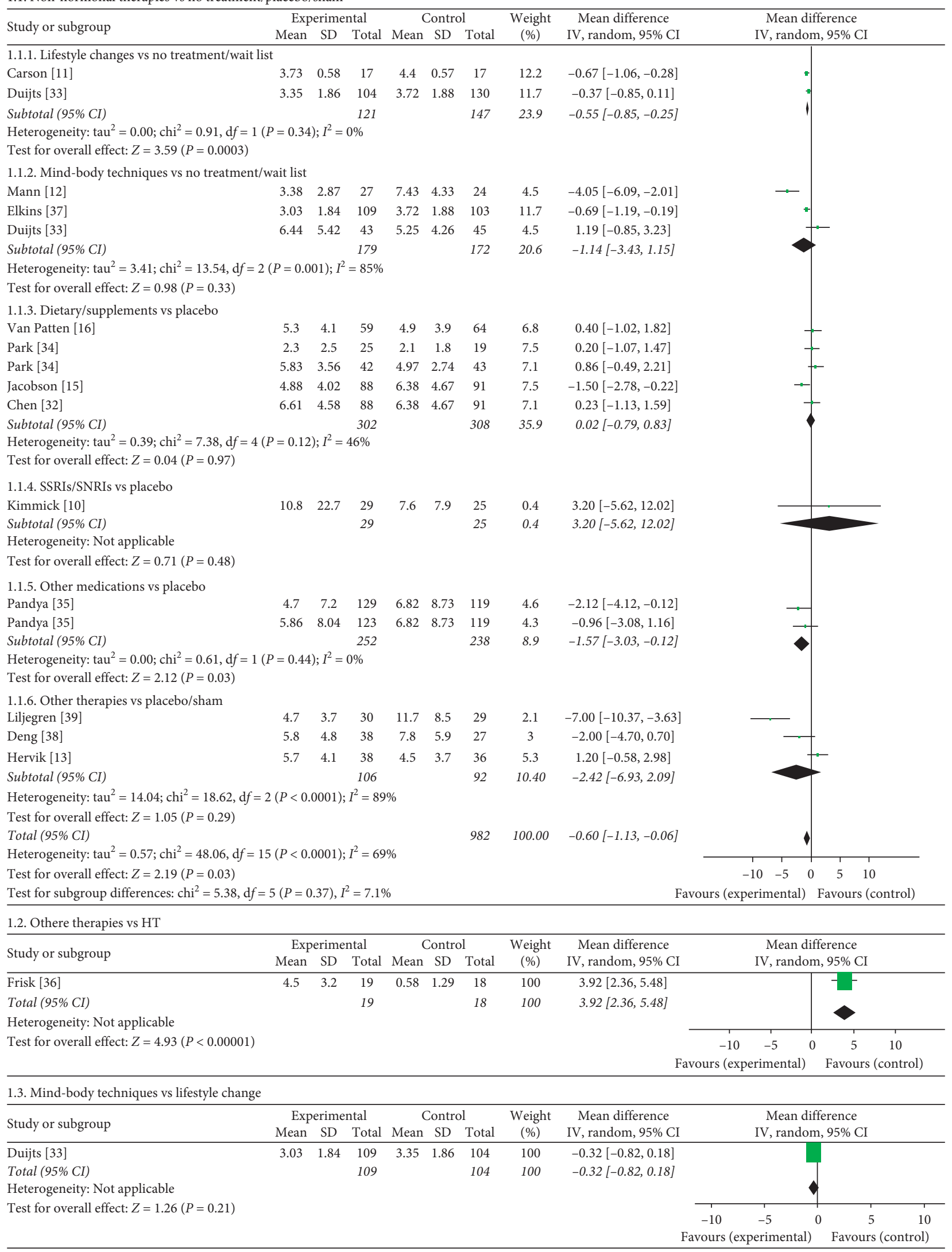

FIGURE 2: Forest plot of hot flash frequency: "any intervention that includes nonhormonal management" vs. "any intervention that does not include nonhormonal management." CI: confidence interval; IV: inverse variance; SD: standard deviation; Std. mean difference: standardized mean difference. 


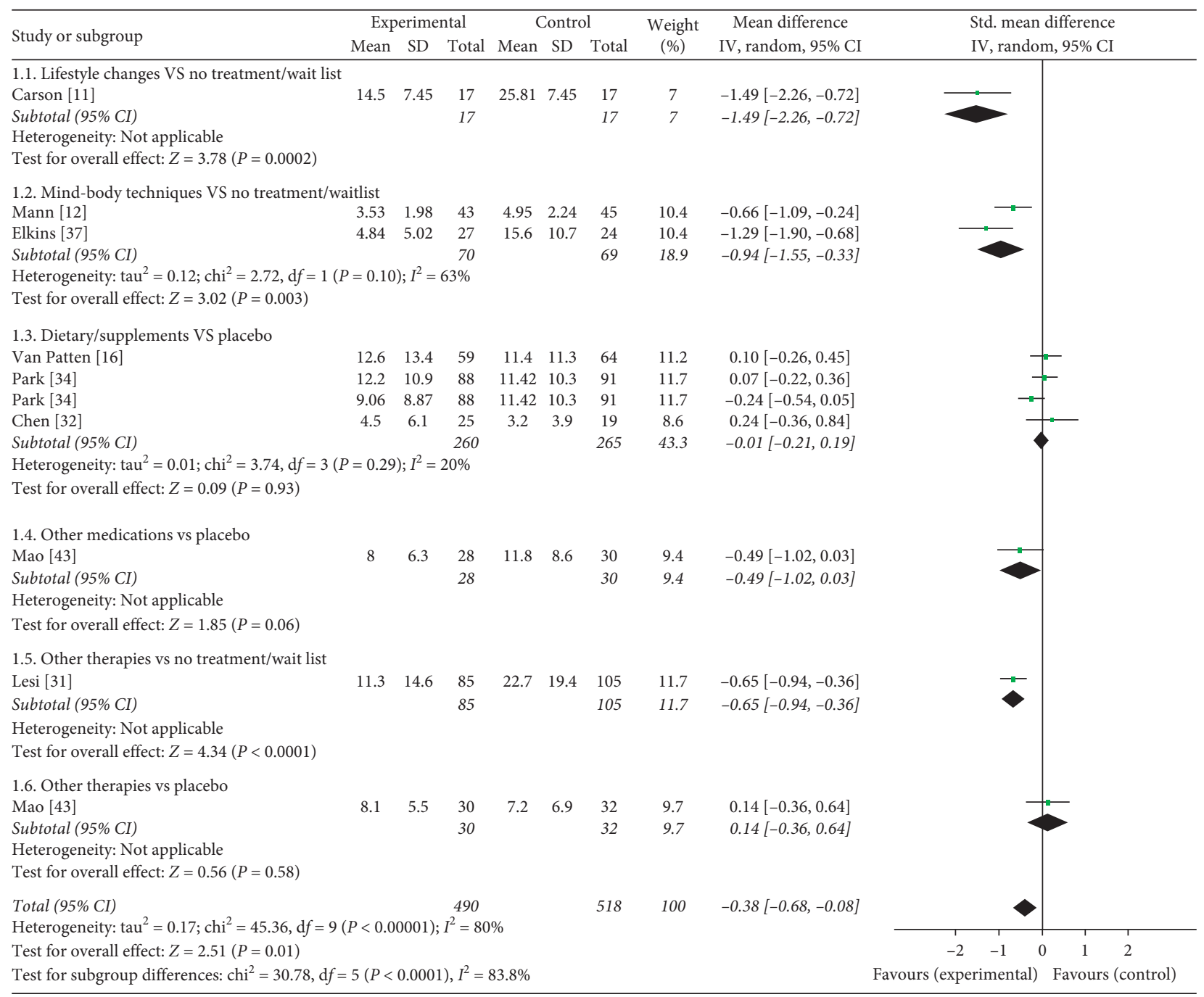

Figure 3: Forest plot of hot flash score: "any intervention that includes nonhormonal management" vs. "any intervention that does not include nonhormonal management." CI: confidence interval; IV: inverse variance; SD: standard deviation; Std. mean difference: standardized mean difference.

3 RCTs $[14,31,38]$ reported adverse events related to acupuncture such as muscle pain and bruising.

3.11. Small-Sample Effect Detection. A comparison-correction funnel plot of hot flash frequency was constructed for the 15 included studies (involving 9 interventions) as shown in Supplementary Material 4. The funnel plot was asymmetrical, and 5 studies fell outside its boundaries, indicating that there might have been a small-sample effect in this study.

\section{Discussion}

4.1. Summary of Evidence. In this network meta-analysis, we assessed the clinical effectiveness and safety of currently available nonhormonal hot flash therapies for breast cancer survivors. We found that acupuncture was widely used in the included studies and was also the highest-ranking nonhormonal therapy for reducing hot flash frequency and score. Experimental data suggests that acupuncture reduces hot flashes. It also seems to increase central endorphin activity which may make thermoregulation more stable and in turn decrease vasomotor symptoms [40, 41]. Most clinical evidence indicates that acupuncture reduces hot flashes more than sham control groups $[42,43]$. This is consistent with our results. Furthermore, our network meta-analysis also showed that placebo ranked higher than treatments such as lifestyle changes, dietary/supplements, and mindbody techniques. Some clinical evidence was consistent with these results $[16,32]$. Therefore, we think that there is a high placebo response rate during hot flash treatment $[44,45]$. SSRIs/SNRIs ranked lower than all other treatments except no treatment/waitlist. As antidepressants, SSRIs/SNRIs may not be fit for hot flash treatment [46]. The safety analysis showed that there had been a few related adverse events during acupuncture treatment and that drug related adverse reactions could have also occurred in studies using drug interventions. Overall, the confidence level for this review was limited. Thus, no confirmative recommendations of nonhormonal therapies for reducing hot flashes in patients with breast cancer can be made at present. Acupuncture may 


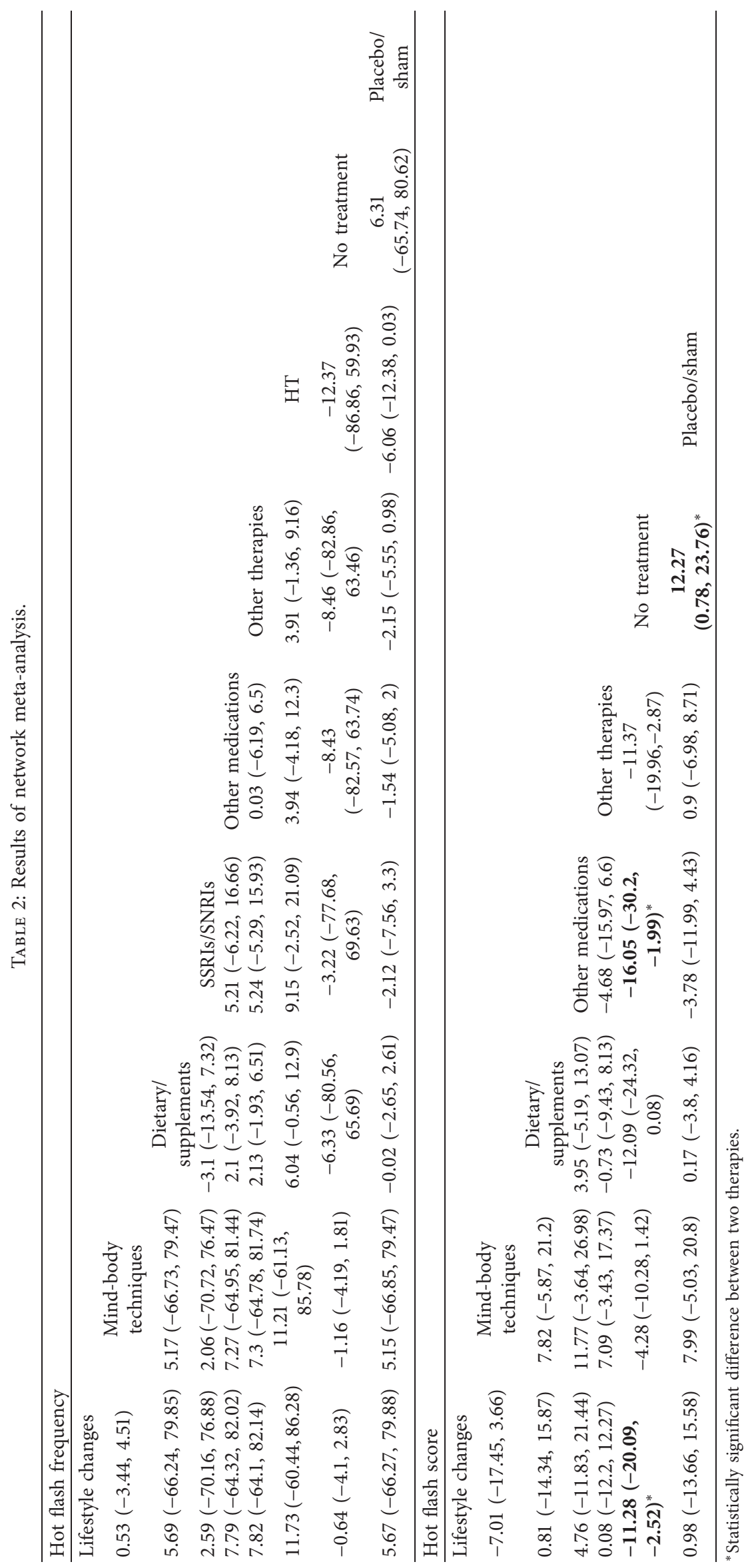




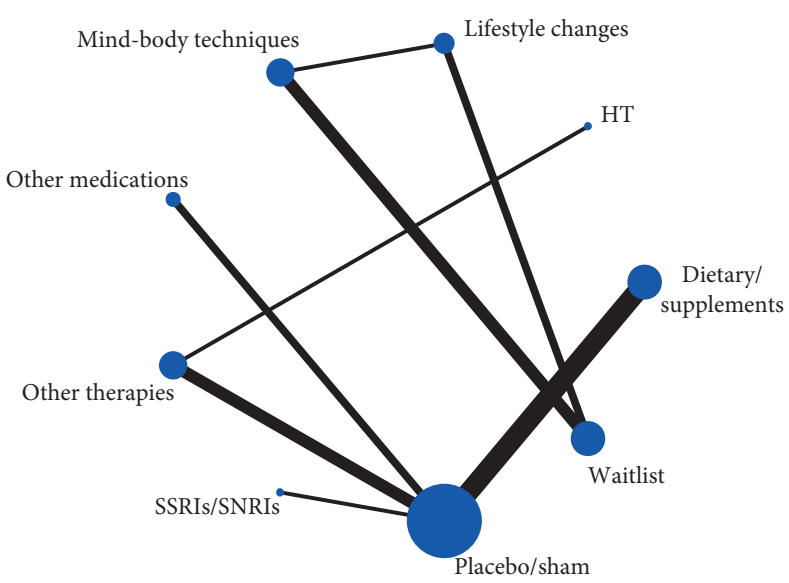

(a)

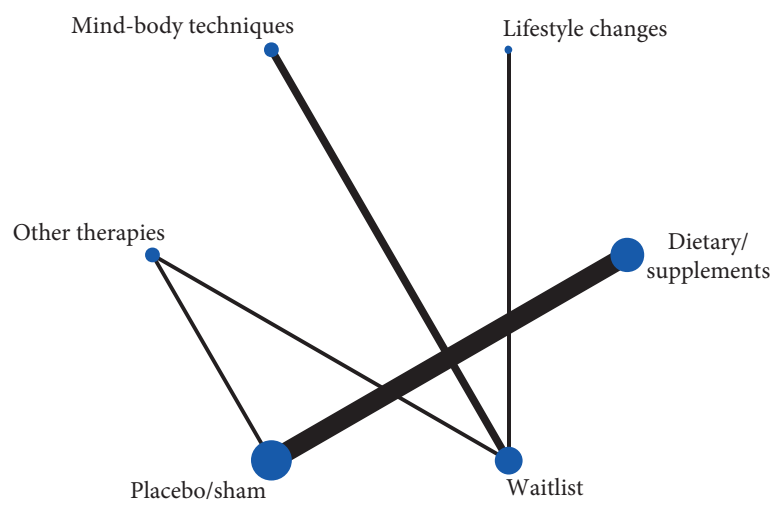

(b)

FIGURE 4: Network plot: (a) hot flash frequency; (b) hot flash score.

Table 3: Ranking of interventions and comparisons.

\begin{tabular}{|c|c|c|c|c|}
\hline \multirow{2}{*}{ Interventions } & \multicolumn{2}{|c|}{ Hot flash frequency } & \multicolumn{2}{|c|}{ Hot flash score } \\
\hline & WinBUGS results & Ranking & WinBUGS results & Ranking \\
\hline Lifestyle changes & 0.150 & 7 & 0.0071 & 3 \\
\hline Mind-body techniques & 0.071 & 6 & 0.064 & 6 \\
\hline Dietary/supplements & 0.066 & 5 & 0.018 & 5 \\
\hline SSRIs/SNRIs & 0.327 & 9 & - & - \\
\hline Other medications & 0.029 & 3 & 0.009 & 4 \\
\hline Other therapies (acupuncture) & 0.007 & 2 & 0.003 & 1 \\
\hline HT & 0.003 & 1 & - & - \\
\hline Placebo/sham & 0.047 & 4 & 0.006 & 2 \\
\hline No treatment/waitlist & 0.300 & 8 & 0.894 & 7 \\
\hline
\end{tabular}

be effective, but more research is needed to determine whether it should be recommended.

4.2. Strengths and Limitations. This research has several strengths. First, this is the first network meta-analysis to investigate the effects and side effects of nonhormonal hot flash therapies for breast cancer survivors. Second, an extensive and sensitive search strategy was used to retrieve as many relevant studies as possible in this systematic review. Third, we conducted a preliminary network meta-analysis for pairwise comparison between the interventions identified. We identified many treatment options, but few studies that directly compared their effectiveness. Under these circumstances, network meta-analysis can provide indirect and mixed effect estimates that allow for comparison of the effects of different interventions [47]. The results of the indirect pairwise comparison give some insight into the potential effectiveness of each intervention, although our network meta-analysis had limited value in this study because of the small quantity of randomized controlled trials included and the potential oversight of relevant interventions.

This research also has several limitations. First, this systematic review was limited by the small number of randomized clinical trials comparing hot flash treatments. Few pairs of treatments had been compared in more than one trial. Therefore, we classified specific treatments according to the statement [21] and then did analysis, which may have affected the results and increased limitations for clinical guidance. Second, we failed to evaluate the safety of each therapy, due to limited data in the primary studies. Future trials should report the relative effectiveness and sideeffect profiles of active treatments in order to determine the best clinical management approach. Third, 50\% of studies were assessed as 'unclear' in allocation concealment. As the hot flush frequency and score are patient-reported outcomes, if there is no blinding (participants and personnel), this could cause no blinding on the outcome assessors, which implies the possibility of detection bias and performance bias. Therefore, methodological quality should be paid more attention when blinding is limited due to the nature of some interventions in future studies. In addition, despite the fairly consistent collection of hot flash frequency and score data using hot flash diaries, studies did not consistently report outcomes in comparable formats. This prohibited the pooling of estimates in meta-analysis. There was great variability in how hot flash outcomes were reported statistically, such as means and standard deviations or standard errors, means and 95\% confidence intervals, and medians 
and ranges. Future studies should report hot flash frequency and score results using a common measure, so that their results can be compared directly.

\section{Conclusion}

This network meta-analysis compares nonhormonal management interventions for hot flashes in breast cancer survivors. Our network meta-analysis suggests that acupuncture has been the most frequently tested intervention and might be more effective in improving hot flashes for breast cancer survivors. Pronounced placebo response was also found in hot flash treatment. The evidence of safety for the nonhormonal therapies is also insufficient. Therefore, no confirmative recommendations of nonhormonal management for hot flashes in breast cancer survivors can be made at present. Further research is needed to determine the recommendations that can be made when enough clinical studies of hot flashes have been conducted.

\section{Conflicts of Interest}

The authors declare that there are no conflicts of interest regarding the publication of this paper.

\section{Authors' Contributions}

JL designed the study, conducted the literature search, did quality assessment, analyzed the data, and drafted the manuscript. HYY supervised the study procedure, designed the study, set up and adjusted eligibility criteria, conducted the literature search, and revised the manuscript. ZHW and LML participated in setting up and adjusting eligibility criteria and in interpreting the results. GNN participated in assessing quality and adjudicating disagreements in study selection and also helped draft the manuscript. YL and DDC participated in study selection. LX and YFL extracted the data. All authors read and approved the final manuscript.

\section{Acknowledgments}

The authors thank Shaonan Liu, Jinhong Zuo, and Geng Li for their contributions to the study. They also thank Benjamin Ross for the language polishing work. This work was funded by the Science and Technology Program of Guangdong Province (No. 2014A020221067) and the Specific Research Fund for TCM Science and Technology of Guangdong Provincial Hospital of Chinese Medicine (Nos. YN2016QL03 and YN2015MS14).

\section{Supplementary Materials}

Supplementary Material 1: search strategies for RCTs on nonhormonal management for breast cancer survivors in PubMed. Supplementary Material 2: risk of bias graph. Supplementary Material 3: the figure of inconsistency assessment for the outcome of hot flash frequency. Supplementary Material 4: the funnel plot of comparison, "any nonhormonal intervention versus no nonhormonal intervention," for the outcome of hot flash frequency. (Supplementary Materials)

\section{References}

[1] C. DeSantis, J. Ma, L. Bryan, and A. Jemal, "Breast cancer statistics, 2013," CA: A Cancer Journal for Clinicians, vol. 64, no. 1, pp. 52-62, 2014.

[2] B. J. Caan, J. A. Emond, H. I. Su et al., "Effect of postdiagnosis weight change on hot flash status among early-stage breast cancer survivors," Journal of Clinical Oncology, vol. 30, no. 13, pp. 1492-1497, 2012.

[3] R. T. Chlebowski, J. E. Mortimer, C. J. Crandall et al., "Persistent vasomotor symptoms and breast cancer in the Women's Health Initiative," Journal of Clinical Oncology, vol. 36, no. 15, Article ID e13567, 2018.

[4] H. I. Su, M. D. Sammel, E. Springer, E. W. Freeman, A. DeMichele, and J. J. Mao, "Weight gain is associated with increased risk of hot flashes in breast cancer survivors on aromatase inhibitors," Breast Cancer Research and Treatment, vol. 124, no. 1, pp. 205-211, 2010.

[5] R. R. Villar, S. P. Fernandez, C. C. Garea, M. T. S. Pillado, V. B. Barreiro, and C. G. Martin, "Quality of life and anxiety in women with breast cancer before and after treatment," Revista Latino-Americana de Enfermagem, vol. 2017, no. 25, Article ID 29267541, 13 pages, 2017.

[6] L. Morales, S. Pans, K. Verschueren et al., "Prospective study to assess short-term intra-articular and tenosynovial changes in the aromatase inhibitor-associated arthralgia syndrome," Journal of Clinical Oncology, vol. 26, no. 19, pp. 3147-3152, 2008.

[7] J. L. Neven, C. M. Saunders, L. I. Emery, H. Green, D. A. Doherty, and M. Hickey, "Nature and severity of menopausal symptoms and their impact on quality of life and sexual function in cancer survivors compared with women without a cancer history," Menopause, vol. 21, no. 3, pp. 267-274, 2014.

[8] Committee on Practice Bulletins-Gynecology, "ACOG Practice Bulletin No. 126: management of gynecologic issues in women with breast cancer," Obstetrics and Gynecology, vol. 119, no. 3, pp. 666-682, 2012.

[9] D. L. Barton, B. I. LaVasseur, J. A. Sloan et al., "Phase III, placebo-controlled trial of three doses of citalopram for the treatment of hot flashes: NCCTG trial N05C9," Journal of Clinical Oncology, vol. 28, no. 20, pp. 3278-3283, 2010.

[10] G. G. Kimmick, J. Lovato, R. McQuellon, E. Robinson, and H. B. Muss, "Randomized, double-blind, placebo-controlled, crossover study of sertraline (Zoloft) for the treatment of hot flashes in women with early stage breast cancer taking tamoxifen," The Breast Journal, vol. 12, no. 2, pp. 114-122, 2006.

[11] J. W. Carson, K. M. Carson, L. S. Porter, F. J. Keefe, and V. L. Seewaldt, "Yoga of Awareness program for menopausal symptoms in breast cancer survivors: results from a randomized trial," Supportive Care in Cancer, vol. 17, no. 10, pp. 1301-1309, 2009.

[12] E. Mann, M. J. Smith, J. Hellier et al., "Cognitive behavioural treatment for women who have menopausal symptoms after breast cancer treatment (MENOS 1): a randomised controlled trial," The Lancet Oncology, vol. 13, no. 3, pp. 309-318, 2012.

[13] J. Hervik and O. Mjåland, "Acupuncture for the treatment of hot flashes in breast cancer patients, a randomized, controlled trial," Breast Cancer Research and Treatment, vol. 116, no. 2, pp. 311-316, 2009. 
[14] J. J. Mao, M. A. Bowman, S. X. Xie, D. Bruner, A. DeMichele, and J. T. Farrar, "Electroacupuncture versus gabapentin for hot flashes among breast cancer survivors: a randomized placebo-controlled trial," Journal of Clinical Oncology, vol. 33, no. 31, pp. 3615-3620, 2015.

[15] J. S. Jacobson, A. B. Troxel, J. Evans et al., "Randomized trial of black cohosh for the treatment of hot flashes among women with a history of breast cancer," Journal of Clinical Oncology, vol. 19, no. 10, pp. 2739-2745, 2001.

[16] C. L. Van Patten, "Effect of soy phytoestrogens on hot flashes in postmenopausal women with breast cancer: a randomized, controlled clinical trial," Journal of Clinical Oncology, vol. 20, no. 6, pp. 1449-1455, 2002.

[17] K. McGarry, M. Geary, and V. Gopinath, "Beyond estrogen: treatment options for hot flashes," Clinical Therapeutics, vol. 40, no. 10, pp. 1778-1786, 2018.

[18] S. L'Esperance, S. Frenette, A. Dionne, and J. Y. Dionne, "Pharmacological and non-hormonal treatment of hot flashes in breast cancer survivors: CEPO review and recommendations," Supportive Care in Cancer: Official Journal of the Multinational Association of Supportive Care in Cancer, vol. 21, no. 5, pp. 1461-1474, 2013.

[19] C. L. Loprinzi, J. Sloan, V. Stearns et al., "Newer antidepressants and gabapentin for hot flashes: an individual patient pooled analysis," Journal of Clinical Oncology, vol. 27, no. 17, pp. 2831-2837, 2009.

[20] G. Rada, D. Capurro, T. Pantoja et al., "Non-hormonal interventions for hot flushes in women with a history of breast cancer," The Cochrane Database of Systematic Reviews, vol. 25, Article ID CD004923, 2010.

[21] The North American Menopause Society, "Nonhormonal management of menopause-associated vasomotor symptoms: 2015 position statement of the North American Menopause Society," Menopause, vol. 22, no. 11, pp. 1155-1172, 2015.

[22] C. Johns, S. M. Seav, S. A. Dominick et al., "Informing hot flash treatment decisions for breast cancer survivors: a systematic review of randomized trials comparing active interventions," Breast Cancer Research and Treatment, vol. 156, no. 3, pp. 415-426, 2016.

[23] J. P. A. Ioannidis, "Integration of evidence from multiple meta-analyses: a primer on umbrella reviews, treatment networks and multiple treatments meta-analyses," Canadian Medical Association Journal, vol. 181, no. 8, pp. 488-493, 2009.

[24] F. Catalá-López, A. Tobías, C. Cameron, D. Moher, and B. Hutton, "Network meta-analysis for comparing treatment effects of multiple interventions: an introduction," Rheumatology International, vol. 34, no. 11, pp. 1489-1496, 2014.

[25] B. Hutton, G. Salanti, D. M. Caldwell et al., "The PRISMA extension statement for reporting of systematic reviews incorporating network meta-analyses of health care interventions: checklist and explanations," Annals of Internal Medicine, vol. 162, no. 11, pp. 777-784, 2015.

[26] J. Higgins and J. Thomas, Cochrane handbook for systematic reviews of interventions, Wiley, Hoboken, NJ, USA, 2019, http:// www.cochrane.org/training/cochrane-handbook.

[27] J. P. T. Higgins, G. S. Thompson, J. J. Deeks, and D. G. Altman, "Measuring inconsistency in meta-analyses," The BMJ, vol. 327, no. 7414, pp. 557-560, 2003.

[28] A. E. Ades, M. Sculpher, A. Sutton et al., "Bayesian methods for evidence synthesis in cost-effectiveness analysis," PharmacoEconomics, vol. 24, no. 1, pp. 1-19, 2006.

[29] G. Lu and A. E. Ades, "Combination of direct and indirect evidence in mixed treatment comparisons," Statistics in Medicine, vol. 23, no. 20, pp. 3105-3124, 2004.
[30] S. Dias, A. J. Sutton, A. E. Ades, and N. J. Welton, "Evidence synthesis for decision making 2," Medical Decision Making, vol. 33, no. 5, pp. 607-617, 2013.

[31] G. Lesi, G. Razzini, M. A. Musti et al., "Acupuncture as an integrative approach for the treatment of hot flashes in women with breast cancer: a prospective multicenter randomized controlled trial (AcCliMaT)," Journal of Clinical Oncology, vol. 34, no. 15, pp. 1795-1802, 2016.

[32] W. Y. Chen, A. Giobbie-Hurder, K. Gantman et al., "A randomized, placebo-controlled trial of melatonin on breast cancer survivors: impact on sleep, mood, and hot flashes," Breast Cancer Research and Treatment, vol. 145, no. 2, pp. 381-388, 2014.

[33] S. F. A. Duijts, M. van Beurden, H. S. A. Oldenburg et al., "Efficacy of cognitive behavioral therapy and physical exercise in alleviating treatment-induced menopausal symptoms in patients with breast cancer: results of a randomized, controlled, multicenter trial," Journal of Clinical Oncology, vol. 30, no. 33, pp. 4124-4133, 2012.

[34] H. Park, R. Qin, T. J. Smith et al., "North central cancer treatment group N10C2 (alliance)," Menopause, vol. 22, no. 6, pp. 627-632, 2015.

[35] K. J. Le-Lindqwister, G. R. Morrow, J. A. Roscoe et al., "Gabapentin for hot flashes in 420 women with breast cancer: a randomised double-blind placebo-controlled trial," The Lancet, vol. 366, no. 9488, pp. 818-824, 2005.

[36] J. Frisk, S. Carlhäll, A.-C. Källström, L. Lindh-Åstrand, A. Malmström, and M. Hammar, "Long-term follow-up of acupuncture and hormone therapy on hot flushes in women with breast cancer: a prospective, randomized, controlled multicenter trial," Climacteric, vol. 11, no. 2, pp. 166-174, 2008.

[37] G. Elkins, J. Marcus, V. Stearns et al., "Randomized trial of a hypnosis intervention for treatment of hot flashes among breast cancer survivors," Journal of Clinical Oncology, vol. 26, no. 31, pp. 5022-5026, 2008.

[38] G. Deng, A. J. Vickers, K. S. Yeung et al., "Randomized, controlled trial of acupuncture for the treatment of hot flashes in breast cancer patients," Journal of Clinical Oncology, vol. 25, no. 35, pp. 5584-5590, 2007.

[39] A. Cassileth, P. Gunnarsson, B.-M. Landgren, N. Robéus, H. Johansson, and S. Rotstein, "Reducing vasomotor symptoms with acupuncture in breast cancer patients treated with adjuvant tamoxifen: a randomized controlled trial," Breast Cancer Research and Treatment, vol. 135, no. 3, pp. 791-798, 2012.

[40] A. C. Spetz Holm, J. Frisk, and M. L. Hammar, "Acupuncture as treatment of hot flashes and the possible role of calcitonin gene-related Peptide," Evidence-Based Complementary and Alternative Medicine, vol. 2012, Article ID 22110545, 9 pages, 2012.

[41] P. Li and J. C. Longhurst, "Neural mechanism of electroacupuncture's hypotensive effects," Autonomic Neuroscience: Basic \& Clinical, vol. 157, no. 1-2, pp. 24-30, 2010.

[42] X. P. Wang, D. J. Zhang, X. D. Wei, J. P. Wang, and D. Z. Zhang, "Acupuncture for the relief of hot flashes in breast cancer patients: a systematic review and meta-analysis of randomized controlled trials and observational studies," Journal of Cancer Research and Therapeutics, vol. 14, pp. S600-s608, 2018.

[43] J. J. Mao, R. Leed, M. A. Bowman et al., "Acupuncture for hot flashes: decision making by breast cancer survivors," The Journal of the American Board of Family Medicine, vol. 25, no. 3, pp. 323-332, 2012. 
[44] L. Li, L. Xu, J. Wu, L. Dong, Y. Lv, and Q. Zheng, "Quantitative analysis of placebo response and factors associated with menopausal hot flashes," Menopause, vol. 24, no. 8, pp. 932-937, 2017.

[45] L. Li, L. Xu, J. Wu, L. Dong, S. Zhao, and Q. Zheng, "Comparative efficacy of nonhormonal drugs on menopausal hot flashes," European Journal of Clinical Pharmacology, vol. 72, no. 9, pp. 1051-1058, 2016.

[46] C. Stubbs, L. Mattingly, S. A. Crawford, E. A. Wickersham, J. L. Brockhaus, and L. H. McCarthy, "Do SSRIs and SNRIs reduce the frequency and/or severity of hot flashes in menopausal women," The Journal of the Oklahoma State Medical Association, vol. 110, no. 110, pp. 272-274, 2017.

[47] A. Cipriani, J. P. Higgins, J. R. Geddes, and G. Salanti, "Conceptual and technical challenges in network metaanalysis," Annals of Internal Medicine, vol. 159, no. 2, pp. 130-137, 2013. 